\title{
Generation of Surface Plasmon Resonance and Lossy Mode Resonance by thermal treatment of ITO thin-films
}

\author{
I. Del Villar, C.R. Zamarreño, M. Hernaez, P. Sanchez, F.J. Arregui and I.R. Matias \\ ${ }^{1}$ Electrical and Electronic Engineering Department, Public University of Navarre, \\ Campus Arrosadia s/n, 31006 Pamplona, NA, Spain \\ Corresponding author: ignacio.delvillar@unavarra.es
}

\begin{abstract}
Silicon wafers coated with Indium Tin Oxide (ITO) by application of sputtering technique have been characterized after different post-annealing techniques, showing that this last factor is critical for the quality of the thin-film and for the creation and tuning of both surface plasmon resonances and lossy mode resonances. By adequate selection of the ITO thin-film thickness both resonances can be tracked in the same spectrum, which can be used in sensor and optical communications fields.
\end{abstract}

Keywords: Thin films, Indium Tin Oxide (ITO), optical resonance, sensors

\section{INTRODUCTION}

Indium Tin Oxide (ITO) is a well-known transparent conductive oxide, which finds application in many different fields such as fabrication of heat shields [1], liquid crystal displays and flat panel displays [2], organic light-emitting diodes [3] and solar cells [4]. This material has also been used in the important field of sensors, more specifically in conductimetric gas sensors [5]. More recently, an application has been found within this last research area. It consists of the 
utilization of an ITO thin-film for generation of Surface Plasmon Resonance (SPR) [6, 7]. SPR is based on the excitation of a Surface Plasmon propagating in a thin-film by means of light coupling [8]. As a result, resonance dips are observed in the electromagnetic spectrum. These resonances depend mainly on three parameters: the refractive index of the film media in contact with the thin film, the refractive index of the thin-film and the thickness of the thin-film. Consequently, the applicability of ITO to develop sensing devices based on conductivity changes induced by certain gases expands to the optical domain [9]. Moreover, it becomes a potential substitute of other well-known materials, such as gold or silver, in the development of biosensors, where a highly selective material, such as antibody or enzyme, is deposited on the thin-film and permits to detect the presence of the target molecule [10].

The reason why ITO is not the most used material in SPR sensors is that SPR is a phenomenon closely related with metallic materials, typically silver and gold [11,12]. Both metals permit to obtain resonance dips in the optical spectrum, typically in the range 400-450 nm and 600-800 nm respectively, when excited by light.

However, as it has been mentioned above, a transparent conductive material like ITO has been successfully used for the generation of SPRs [6,7].

Another resonance phenomenon is Lossy Mode Resonance (LMR) [13,14], also named guided mode resonance or $\mathrm{TE}_{0}$ and $\mathrm{TM}_{0}$ resonances by other authors $[15,16]$. This phenomenon occurs in different conditions: for SPRs the real part of the thin-film permittivity is negative and higher in magnitude than both its own imaginary part and the permittivity of the material surrounding the thin-film, whereas for LMRs the real part of the thin-film permittivity must be positive and higher in magnitude than both its own imaginary part and the material surrounding the thin-film 
[15]. Consequently, non-metallic materials are adequate for LMR generation (polymers $[17,18]$ and conducting oxides [19]), and can be used for sensing applications.

Surprisingly, a material like ITO, which is used for generation of an SPR [7], can also be used for LMR generation [13]. This apparent contradiction is explained in detail in this work.

To this purpose several ITO coated silicon wafers are subjected to different thermal posttreatments. According to [20-22], the post-annealing plays a major role in the properties of ITO thin-films. Consequently, the composition, crystallinity, conductivity, contact angle, and refractive index dispersion is studied for all samples in section 2. After the characterization, the optical spectrum in a Kretschmann configuration is analyzed in section 3, showing the possibility to generate both SPR and LMR at the same time. Finally, some concluding remarks are given in the last section.

\section{DEPOSITION AND CHARACTERIZATION OF ITO THIN FILMS}

\section{Experimental}

ITO coatings were deposited with sputtering technique onto four polished silicon wafer substrates (P type doped with boron, orientation $\langle 100\rangle$, with polished front surface and etched back surface from Silicon Valley Microelectronics Inc.).

Prior to the deposition of the ITO coating, it is necessary to prepare the substrates by following a cleaning procedure using acetone, methanol and isopropanol. After that, for the deposition of the ITO coatings, a sputtering equipment (K675XD from Quorum Technologies, Ltd.) was used with a partial pressure of argon of $8 \times 10^{-3} \mathrm{mbar}$ and a current intensity of $150 \mathrm{~mA}$.

Three of the samples (A, B, and C) were thermally post-annealed at $500{ }^{\circ} \mathrm{C}$ for 4 hours in a controlled atmosphere oven (Linn High Therm GmbH VMK-39). Sample A was annealed under 
vacuum atmosphere, sample B under nitrogen atmosphere, and sample $\mathrm{C}$ under air atmosphere. The thermal treatment of samples $\mathrm{A}, \mathrm{B}$ and $\mathrm{C}$ finished with a cooling process at room temperature. Sample D was not subjected to thermal treatment.

After that, the thickness, the composition, the crystallinity, the contact angle and the refractive index dispersion of the ITO thin-films were analyzed. To this purpose a field emission scanning electron microscope (SEM) UltraPlus, from Carl Zeiss Inc with an in-lens detector at $3 \mathrm{kV}$ and an aperture diameter of $30 \mu \mathrm{m}$, an X-ray diffractometer (XRD) SIEMENS D 5000 (angle of incidence $1^{\circ}$ ), an atomic force microscope (AFM) model diInnova from Veeco in tapping mode and with a scanner of $50 \times 50 \mu \mathrm{m}$, a CCD camera based instrument for measuring contact angles (CA) of liquids on solids model CAM100 from KSV (the liquid: used in the experiments is ultrapure deionized water 18.2Mohm and the volume used for each measurement 5 microliters), and an ellipsometer UVISEL with spectral range 0.6-6.5 eV $(190-2100 \mathrm{~nm})$, an angle of incidence $70^{\circ}$, an spot size $1 \mathrm{~mm}$ and Software DeltaPsi2TM (from Horiba Scientific Thin Film Division) were used.

\section{Characterization of ITO thin-films}

For the sake of comparison all samples were ITO sputtered for the same time, which should lead to the same coating thickness in all silicon wafers. The results obtained with the scanning electron microscope (SEM) (see Fig. 1) confirm this fact. The thickness of the samples is around $80 \mathrm{~nm}$.

In Fig. 2 the XRD pattern of ITO coated silicon wafers can be observed. For sample D no peaks corresponding to X-ray diffraction from planes are observed, whereas for samples A, B and C there are several peaks. This proves that the film is amorphous when no thermal treatment is applied, whereas the opposite is true with a post-annealing. For samples A, B and C the 
predominant peak is located at 31 degrees, corresponding to Miller index (222), which indicates that the grains of the thin-film are predominantly aligned in the (111) direction. The rest of peaks starting from the lowest angle are: (211), (222), (400), (440) and (622) [23].

In Fig. 3 atomic force microscopy (AFM) measurements reveals the roughness of the samples and the crystallinity of the thin-film. Sample B exhibits a larger grain structure than the rest of samples, which is typical in polycrystalline thin films [24]. Besides, the roughness, expressed as the root mean square (RMS) of the peak heights, is $0.2169,0.6903,0.1720$ and $0.1156 \mathrm{~nm}$ for samples A, B, C and D respectively.

Another important parameter is the conductivity. In this sense it has been calculated the resistivity of the samples $(\Omega / \mathrm{cm})$. The values obtained are $448,160,393$ and 1311 for samples A, B, C and D respectively. Sample B, the one with nitrogen atmosphere post-annealing, shows the highest conductivity. This is explained by the larger grain size observed in Fig. 3, which means a lower grain boundary scattering, leading to an increase in the conductivity [24]. The reason for this lower grain boundary scattering is explained in [25], where it is proved for $(\mathrm{Ba}, \mathrm{Sr}) \mathrm{TiO}_{3}$ films that post-annealing in oxygen atmosphere, contrary to post-annealing in nitrogen atmosphere, leads to the presence of both air to oxygen vacancies and negatively charged oxygen, trapped at the grain boundary at the electrode/dielectric films interface. This idea is confirmed in [26] for ITO thin-films.

Finally, sample D, with no thermal treatment, is the least conductive, which is logical in view of its amorphous structure observed with the XRD pattern obtained in Fig. 2. This confirms that thermal post-annealing improves the conductivity of the thin film [7].

Regarding the contact angle, the results of samples A, B, C with angles 66.9, 65.8, 67.9 respectively are very similar. These values are in agreement to the contact angle of commercial 
ITO [27,28], and are dissimilar to the contact angle of sample D, 87.3. The explanation for this difference is that samples $\mathrm{A}, \mathrm{B}$ and $\mathrm{C}$ are thermally treated, which causes changes in the morphology of a metal oxide thin-film [29], and sample D is not thermally treated.

In Fig. 4 ellipsometric measurements are presented of the complex refractive index (n, k) of the indium tin oxide (ITO) layer.

Plots for sample B and C are similar to each other and resemble that of [20]. If data are modeled with modified Drude free-electron model [7]:

$\varepsilon(\omega)=\varepsilon_{\infty}-\frac{\omega_{p}^{2}}{\omega^{2}+i \omega \Gamma}$

where $\omega_{p}$ is the plasma frequency, $\varepsilon_{\infty}$ is the infrared dielectric constant and $\Gamma$ is the damping coefficient, the plasma frequency of samples $\mathrm{B}$ and $\mathrm{C}$ is respectively $1.731 \times 10^{15} \mathrm{rad} / \mathrm{s}$ and $1.603 \times 10^{15} \mathrm{rad} / \mathrm{s}$. Sample B is more closely related to the value obtained in [20]: $1.89 \times 10^{15} \mathrm{rad} / \mathrm{s}$. On the other hand, plots for sample A are middle-way between those of [20] and those of references [1,7]. The plasma frequency for sample A is $2.131 \times 10^{15} \mathrm{rad} / \mathrm{s}$, whereas for [7] it is $2.693 \times 10^{15} \mathrm{rad} / \mathrm{s}$ and for [1] it is $3.33 \times 10^{15} \mathrm{rad} / \mathrm{s}$.

The plots obtained for sample D do not resemble those typical of ITO. The shape of the plot representing the real part of the refractive index is flat. The same occurs for the imaginary part. Consequently, no plasma frequency can be found, which is closely related to the low conductivity indicated before for sample D. However, the conductivity does not permit to determine the wavelength ranges regions where SPR and LMR can be generated. For SPR it is necessary to know the plasma frequency [6], which depends on the dispersion curves of ITO (Fig. 4). However, a simpler way to know the regions where SPRs can be obtained without the need for a dispersion model, is by following the rules indicated in [15], which were indicated in the introduction: the real part of the thin-film permittivity is negative and higher in magnitude 
than both its own imaginary part and the permittivity of the material surrounding the thin-film. This region is located from $1962 \mathrm{~nm}$ to higher wavelengths for sample A (this is indicated in Fig. 4 with a discontinuous line) and it is located above $2066 \mathrm{~nm}$ (beyond the wavelength range explored in the ellipsometric measurements) for samples B and C.

Also in [15] the rules for generation of LMRs are given: the real part of the thin-film permittivity must be positive and higher in magnitude than both its own imaginary part and the material surrounding the thin-film. Consequently, LMRs can be generated for sample A at wavelengths lower than $1517 \mathrm{~nm}$ (this is indicated in Fig. 4 with a discontinuous line), for sample B at wavelengths lower than $1861 \mathrm{~nm}$ and for sample C at wavelengths lower than $2006 \mathrm{~nm}$. In view of these results, it can be concluded that both the LMR wavelength region and the SPR region are closely related. If the limit of the SPR region is red shifted, the limit of the LMR region increases its wavelength (the opposite is true if the limit of the SPR region is blue shifted).

To conclude this section, after the characterization of the ITO thin-films it is clear that the key parameter for determining the position of the SPR or the LMR is the refractive index dispersion. Consequently, in the next section focus will be centered on analyzing the optical spectra with a Kretschmann configuration, which is mainly dependent of this parameter.

\section{ANALYSIS OF THE OPTICAL SPECTRUM WITH A KRETSCHMANN CONFIGURATION}

In order to study the possibility of generating an SPR and an LMR with the ITO thin-films of section 2, a Kretschmann configuration analyzed with a numerical method based on plane wave propagation is used [30]. The refractive index of the input medium is that of silica [31], and the refractive index of the output medium is that of air. 
In view that, contrary to SPRs, LMRs can be obtained for both TE polarization and TM polarization [32], the reflectance for both polarizations is considered: $R_{\mathrm{TE}}(\theta, \lambda)$ and $\mathrm{R}_{\mathrm{TM}}(\theta, \lambda)$ Again the influence of the thermal post-annealing on the properties of the resonances will be analyzed by comparing the samples. However, for the sake of simplicity only two samples will be considered: sample A and sample B. Sample C is discarded because it shows dispersion curves similar to sample B. On the other hand, it was proved in section 2 that $\mathrm{D}$ sample shows dispersion curves not typical of ITO. Consequently it is not adequate for the analysis of this section

It is also important to indicate that the thickness of the samples in section II (see Fig. 1), was not randomly selected. The objective was a thickness of $80 \mathrm{~nm}$ because an SPR is typically obtained for values in the range 10-200 $\mathrm{nm}$ [7], and an LMR can be obtained for higher values [33], but its position in the optical in the optical spectrum is highly influenced by this parameter (it experiments a progressive wavelength shift to the red as a function of thickness and for low values it is located at wavelengths below the spectrum analyzed). Consequently, a trade-off was chosen between the need for lower thin-film thickness in SPR generation and higher thin-film thickness in LMR generation. In the simulation results (SR) shown below it will be proved that a thickness of $80 \mathrm{~nm}$ permits to visualize both LMRs and SPRs in the spectrum from 350 to 2000 nm.

First, the reflectance for the sample A is analyzed. In Fig. 5a, SR for sample A at TE polarization for angles below TIR $\left(0-42^{\circ}\right)$ are presented.

For low incidence angles one minimum and one maximum are observed in the optical spectrum at 370 and $590 \mathrm{~nm}$ respectively. The explanation is that the thin-film is actually a Fabry-Pérot cavity for angles approaching normal incidence. For the minimum at $370 \mathrm{~nm}$ the refractive index 
of sample $\mathrm{A}$ is 2.11. If these values and a thickness of $80 \mathrm{~nm}$ are introduced in the expression of the phase shift of the optical beam in the cavity formed by the ITO coating:

$\phi=\frac{4 n d \pi}{\lambda}$

where $d$ is the cavity length, $n$ is the refractive index of the coating and $\lambda$ is the wavelength, the phase shift approximates $2 \pi$, which means a constructive interference in transmission and a destructive interference in reflection.

Regarding the maximum at $590 \mathrm{~nm}$, the refractive index of sample A at this wavelength is 1.92 . If these values are introduced in expression (2), the phase shift is $\pi$ (destructive interference in transmission and constructive in reflection). Both the minimum at $370 \mathrm{~nm}$ and the maximum at $590 \mathrm{~nm}$ can be observed also for TM polarization in Fig. 6a. However, the other minimum at $1400 \mathrm{~nm}$ for TE polarization is not observed for TM polarization because it is not due to the Fabry-Pérot interferometer (the refractive index of sample A at this wavelength is lower than that of silica).

As well as the critical angle is approached, the interferometry is progressively reduced. For angles above $\operatorname{TIR}\left(44-90^{\circ}\right)$, the reflection is maximum except for angles approaching $90^{\circ}$, where a dip and thin resonance occurs around $800 \mathrm{~nm}$ (see Fig. 5b). This is a lossy mode resonance (LMR), which is obtained by coupling to a TE lossy mode in the ITO thin-film [13].

In Fig. 6, where SR for TM polarization are presented, the main difference with TE polarization is that in the proximity of the critical angle a double resonance is created, which for angles above TIR (see Fig. 5b) becomes a single resonance at wavelengths above $1500 \mathrm{~nm}$. In view of its only presence for TM polarization, it is a surface Plasmon resonance (SPR). This is corroborated with the results of [6,7], where the SPR occurs for angles between 40 and $60^{\circ}$ and wavelengths higher than $1100 \mathrm{~nm}$. This value changes depending on the ITO properties. For example in [35] it is 
stated that, with an ITO thin-film, an SPR can only be obtained for wavelengths higher than 1500 $\mathrm{nm}$. However, even though there is a variation of the exact limit, the resonance obtained at 600 $\mathrm{nm}$ in Fig. $6 \mathrm{~b}$ cannot be an SPR, especially considering the non-metallic behavior of ITO samples at this wavelength in Fig. 4. This is actually another LMR, obtained by coupling of light to a TM lossy mode. The position in the optical spectrum is different to that of the LMR generated in Fig. 5b for TE polarized light. This is explained by the difference in the effective indices of TE and TM lossy modes [13].

Another interesting question is that the LMR obtained for TE polarization (see Fig. 5b) is much deeper that the LMR obtained for TM polarization (see Fig. 6b). However, it is important to indicate that angles in steps of $1^{\circ}$ have been analyzed. The depth of the resonance is a very sensitive parameter [32], and it would be necessary to sweep in steps lower than $1^{\circ}$ to see the maximum depth that can be achieved. In fact in Fig. $7 \mathrm{~b}$ the resonance obtained for sample B at TE polarization is deeper than that obtained in Fig. $5 \mathrm{~b}$ for sample A.

For sample B the same analysis is done both for TE (see Fig. 7) and TM polarization (see Fig. 8). Conclusions are similar to those obtained for sample A. The main difference is the position of the SPR in Fig. 8b. It is shifted from a wavelength of $1900 \mathrm{~nm}$ (see Fig 6b), to more than 2000 $\mathrm{nm}$, which agrees with the position of SPR limits indicated at the end of section 2. The position of the LMR at TE polarization is also different. For sample A it was located below $800 \mathrm{~nm}$, whereas for sample B it is located above $800 \mathrm{~nm}$. The same is true for the LMR obtained at TM polarization: for sample A it is located below $555 \mathrm{~nm}$ and for sample B it is located above 555 $\mathrm{nm}$.

This proves that it is possible to tune the regions of generation of SPRs and LMRs by changing the thermal treatment. As an example of a possible application, if the devices analyzed in this 
section are used as optical filters, their resonance wavelength can be tuned by adequate selection of the thermal post-treatement, which is interesting in terms of adaptation to specific optical communication windows, for instance.

Another application is optical sensing, where the operating wavelength can be moved to the central wavelength of a laser if a device is based on this type of optical source.

\section{CONCLUSIONS}

To conclude, the application of a specific thermal treatment is critical for obtaining ITO films with different physical properties such as distinctive dispersion curves. It has been proved that, when no thermal treatment is applied, the thin-film is amorphous, whereas the opposite is true for post-annealed structures. Moreover, when no thermal treatment is applied, the refractive index dispersion curves indicate that it is not possible to obtain a resonance in the optical spectrum. In fact the refractive index the key parameter that permits to control the generation and control of a resonances in the optical spectrum

The selection of a thermal treatment under vacuum, nitrogen or air atmosphere permits to modify the refractive index dispersion and, consequently, to tune the wavelength region where resonances can be obtained. In this sense, it has been shown that ITO is an interesting material, which allows generating two different different types of resonances: the well-known surface plasmon resonance (SPR) and the less explored lossy mode resonance (LMR).

It has been also clarified that an adequate selection of the ITO film thickness enables the generation of both types of resonances in the spectral range 450-2000 nm. In other words, the results in articles reporting that ITO can be used for SPR generation $[6,7,9]$, and the results in articles reporting that ITO can be used for LMR generation [13,32,34], are not contradictory. An 
SPR is obtained in a wavelength region where ITO owns a metallic behavior, and LMR is obtained in a wavelength region where ITO owns a non-metallic behavior.

By modifying the thermal post-annealing it is possible to fit the wavelength range of resonances obtained with ITO thin-film based devices according to the optical equipment of the specific application the device is designed for. This could be used for the development of interesting applications, such as sensors and optical filters.

\section{ACKNOWLEDGMENTS}

This work was supported in part by the Spanish Ministry of Education and Science-FEDER TEC2013-43679-R. Special thanks to the Department of Physics of the Public University of Navarra for the utilization of the X-ray diffractometer, to FIDENA S.A. for the utilization of the

SEM and to Horiba Scientific, Thin Film Division for the spectrometric ellipsometry characterization of the samples. 


\section{REFERENCES}

1. I. Hamberg, A. Hjortsberg, and C. G. Granqvist, "High quality transparent heat reflectors of reactively evaporated indium tin oxide," Appl. Phys. Lett., 40 (1982) 362-364.

2. J. E. Costellamo, "Handbook of Display Technology" (Academic Press, New York, 1992).

3. H. Chen, C. Qiu, M. Wong, and H. S. Kwok, "DC Sputtered Indium-Tin Oxide Transparent Cathode for Organic Light-Emitting Diode”, IEEE Electron. Device Lett., 24 (2003) 315317.

4. L. Zhao, Z. Zhou, Hua Peng, R. Cui, "Indium tin oxide thin films by bias magnetron rf sputtering for heterojunction solar cells application," Appl. Surf. Sci., 252 (2005) 385-392.

5. N. G. Patel, P. D. Patel and V. S. Vaishnav, "Indium tin oxide (ITO) thin film gas sensor for detection of methanol at room temperature," Sens. Actuators B, 96 (2003) 180-189.

6. C. Rhodes, S. Franzen J. P. Maria, M. Losego, D. N. Leonard, B. Laughlin, G. Duscher, and S. Weibel, "Surface plasmon resonance in conducting metal oxides," J. App. Phys., 100 (2006) 054905 - 054905-4.

7. C. Rhodes, M. Cerruti, A. Efremenko, M. Losego, D. E. Aspnes, J. P. Maria and S. Franzen, "Dependence of plasmon polaritons on the thickness of indium tin oxide thin films," J. App. Phys., 103 (2008) 093108 - 093108-6.

8. J. Homola, "Surface Plasmon Resonance Based Sensors” pp. 26-43, (Springer, 2006).

9. S.K. Mishra, B.D. Gupta, "Surface Plasmon Resonance-Based Fiber-Optic Hydrogen Gas Sensor Utilizing Indium-Tin Oxide (ITO) Thin Films,” Plasmonics, 7 (2012) 627-632.

10. J. Homola, "Surface plasmon resonance sensors for detection of chemical and biological species", Chem. Rev., 108 (2008) 462-493. 
11. K. C. Lee, S. J. Lin, C. H. Lin, C. S. Tsai, and Y. J. Lu, "Size effect of Ag nanoparticles on surface plasmon resonance,” Surf. Coat. Technol. 202 (2008) 5339-5342.

12. X. Zhang, J. Zhang, H. Wang, Y. Hao, X. Zhang, T. Wang, Y. Wang, R. Zhao, H. Zhang, and B. Yang, "Thermal-induced surface plasmon band shift of gold nanoparticle monolayer: Morphology and refractive index sensitivity” Nanotechnology, 21 (2010) 4657029.

13. I. Del Villar, C. R. Zamarreño, M. Hernaez, F. J. Arregui, I. R. Matias, "Lossy mode resonance generation with indium tin oxide coated optical fibers for sensing applications," $J$. Lightwave Technol., 28 (2010) 111-117.

14. F. J. Arregui, I. Del Villar, J. M. Corres, J. Goicoechea, C. R. Zamarreño, C. Elosua, M. Hernaez, P. J. Rivero, A. B. Socorro, A. Urrutia, P. Sanchez, P. Zubiate, D. Lopez, N. De Acha, I. R. Matias, “Fiber-Optic Lossy Mode Resonance Sensors,” Plenary talk, in Eurosensors, Brescia, Italy, 2014.

15. F. Yang and J. R. Sambles, "Determination of the optical permittivity and thickness of absorbing films using long range modes," J. Mod. Opt., 44 (1997) 1155-1163.

16. Y Zhang, "Long-range surface polaritons in thin layers of absorbing materials," M.S. thesis, Eindhoven University of Technology, Netherland, 2011.

17. C. R. Zamarreño, M. Hernáez, I. Del Villar, I. R. Matías and F. J. Arregui “Optical fiber pH sensor based on lossy-mode resonances by means of thin polymeric coatings," Sens. Actuators B, 155 (2011) 290-297.

18. A. B. Socorro, J. M. Corres, I. Del Villar, F. J. Arregui and I. R. Matias, "Fiber-optic biosensor based on lossy mode resonances," Sens. Actuators B, 164 (2012) 263-269. 
19. M. Hernaez, I. Del Villar, C. M. Zamarreño, F. J. Arregui, I. R. Matias, “Optical fiber refractometers based on lossy mode resonances supported by $\mathrm{TiO} 2$ coatings" Appl. Opt., 49 (2010) 3980-3985

20. S. Laux, N. Kaiser, A. Zöller, R. Götzelmann, H. Lauth and H. Bernitzki, "Roomtemperature deposition of indium tin oxide thin films with plasma ion-assisted evaporation" Thin Solid Films, 335 (1998) 1-5.

21. G. Gonçalves, E. Elangovan, P. Barquinha, L. Pereira, R. Martins, and E. Fortunato "Influence of post-annealing temperature on the properties exhibited by ITO, IZO and GZO thin films", Thin Solid Films, 515 (2007) 8562-8566.

22. C. Guillen, J. Herrero, "Structure, optical, and electrical properties of indium tin oxide thin films prepared by sputtering at room temperature and annealed in air or nitrogen," J. Appl. Phys., 101 (2007) 073514.

23. C. G. Choi, K. No, W. J. Lee, H. G. Kim, S. O. Jung, W. J. Lee, W. S. Kim, S. J. Kim, and C. Yoon, "Effects of oxygen partial pressure on the microstructure and electrical properties of indium tin oxide by d.c. magnetron sputtering”, Thin Solid Films, 258 (1995) 274-278.

24. D. Kim, S. Kim, "Effect of secondary ion beam energy and oxygen partial pressure on the structural, morphological and optical properties of ITO films prepared by DMIBD technique", Surf. Coat. Technol., 154 (2002) 204-208.

25. F. M. Pontes, E. R. Leite, E. Longo, J. A. Varela, E. B. Araujo and J. A. Eiras, "Effects of the postannealing atmosphere on the dielectric properties of (Ba,Sr)TiO3 capacitors: Evidence of an interfacial space charge layer," Appl. Phys. Lett., 76 (2000) 2433-2435.

26. D. Mergel, W. Stass, G. Ehl, and D. Barthel, "Oxygen incorporation in thin films of In2O3:Sn prepared by radio frequency sputtering," J. Appl. Phys. 88 (2000) 2437-2492. 
27. J. Seol, M. L. Monroe, T. J. Anderson, M. A. Hasnain, and C. Park, “Effect of ITO surface treatment on organic solar cells" Conference Record of the 2006 IEEE 4th World Conference on Photovoltaic Energy Conversion, WCPEC-4 1, (2007) art. no. 4059606, 236

28. R.D. Sun, A. Nakajima, A. Fujishima, T. Watanabe, K. Hashimoto, "Photoinduced Surface Wettability Conversion of ZnO and TiO2 thin films", J. Phys. Chem. B 105, (2001) 19841990

29. Z.Z. You "Combined AFM, XPS, and contact angle studies on treated indium-tin-oxide films for organic light-emitting devices" Mat. Lett. 61, (2007) 3809-3814.

30. P. Yeh, A. Yariv, and C. S. Hong, "Electromagnetic propagation in periodic stratified media. I. General theory," J. Opt. Soc. Am. 67 (1977) 423-438.

31. G. P. Agrawal, "Nonlinear fiber optics” p. 8., (3rd ed., Academic Press: New York, 2001)

32. I. Del Villar, C. R. Zamarreño, M. Hernaez, F. J. Arregui, I. R. Matias, “Generation of Lossy Mode Resonances With Absorbing Thin-Films," J. Lightwave Technol., 28 (2010) 33513357.

33. I. Del Villar, M. Hernáez, C. R. Zamarreño, P. Sanchez, C. Fernandez-Valdivielso, F. J. Arregui and I. R. Matías, "Design Rules for Lossy Mode Resonance Based Sensors”, Appl. Opt. 51(2012) 4298-4307.

34. A. Kumar, V. K. Sharma, D. Kumar, A. Kapoor "Integrated optic TE/TM pass polarizers using resonant coupling between ITO thin film lossy modes and dielectric waveguide modes," Opt. Comm., 291 (2013) 247-252. 


\section{Figure captions}

Fig. 1: Scanning electron microscope (SEM) of ITO coated silicon wafer samples A, B, C and D. Fig. 2: XRD pattern of ITO coated silicon wafer samples A, B, C and D.

Fig. 3: Atomic force microscopy (AFM) measurements of ITO coated silicon wafer samples A, B, C and D.

Fig. 4: Complex refractive index (n, k) dispersion curves of ITO coated silicon wafer samples A, B, C and D.

Fig. 5: Simulation results: reflectance for sample A (TE polarization) as a function of wavelength at incidence angles: a) $0-42^{\circ}$ b) $44-90^{\circ}$

Fig. 6: Simulation results: reflectance for sample A (TM polarization) as a function of wavelength at incidence angles: a) $0-42^{\circ}$ b) $44-90^{\circ}$

Fig. 7: Simulation results: reflectance for sample B (TE polarization) as a function of wavelength at incidence angles: a) $0-42^{\circ}$ b) $44-90^{\circ}$

Fig. 8: Simulation results: reflectance for sample B (TM polarization) as a function of wavelength at incidence angles: a) $0-42^{\circ}$ b) $44-90^{\circ}$ 
Fig. 1
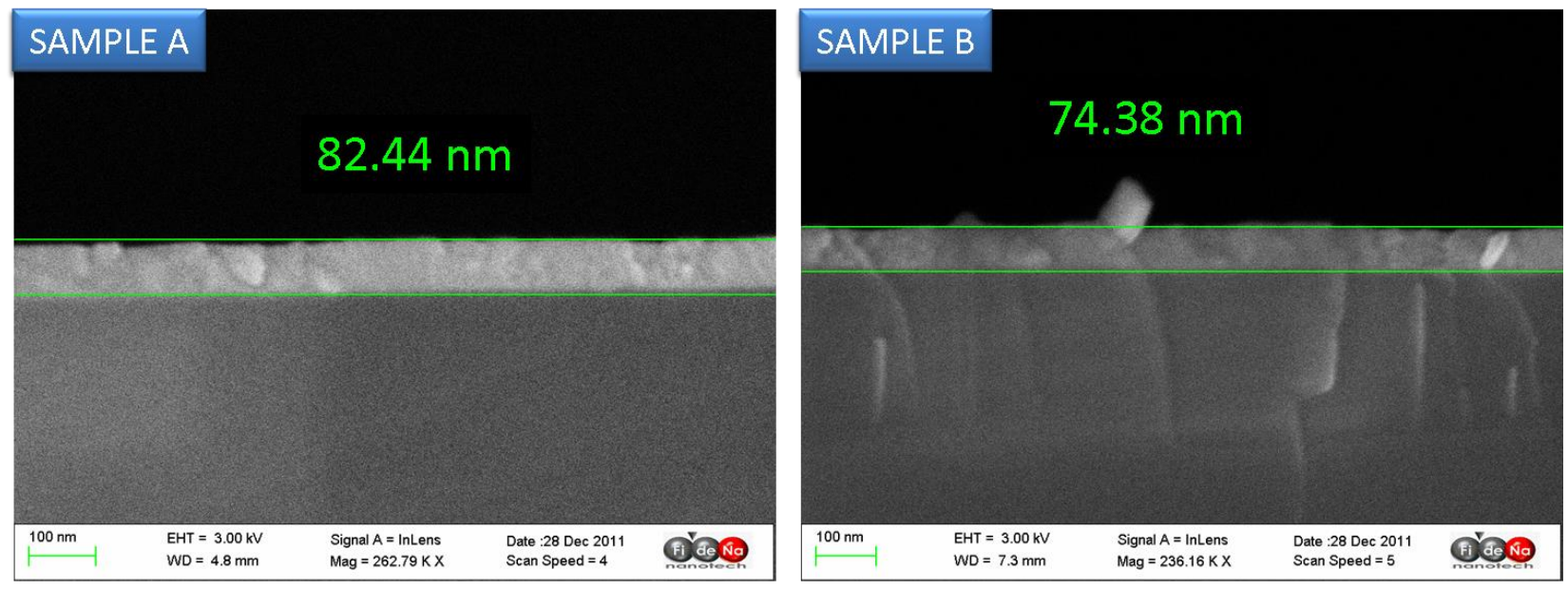

\section{SAMPLE C}

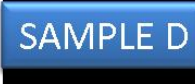

\section{$81.82 \mathrm{~nm}$}

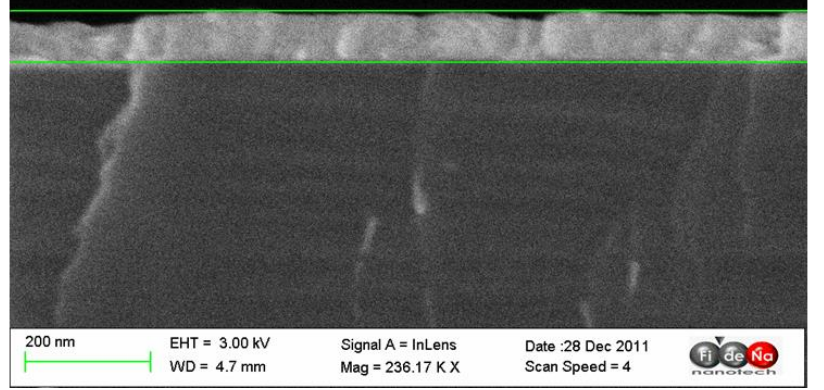

$100 \mathrm{~nm}$

$\mathrm{EHT}=3.00 \mathrm{kV}$ $W D=4.6 \mathrm{~mm}$

Signal $A=\operatorname{lnLens}$

Date :28 Dec 201 Scan Speed $=5$

\section{$87.06 \mathrm{~nm}$}


Fig. 2

Incidencia Rasante. $\theta=1^{\circ}$

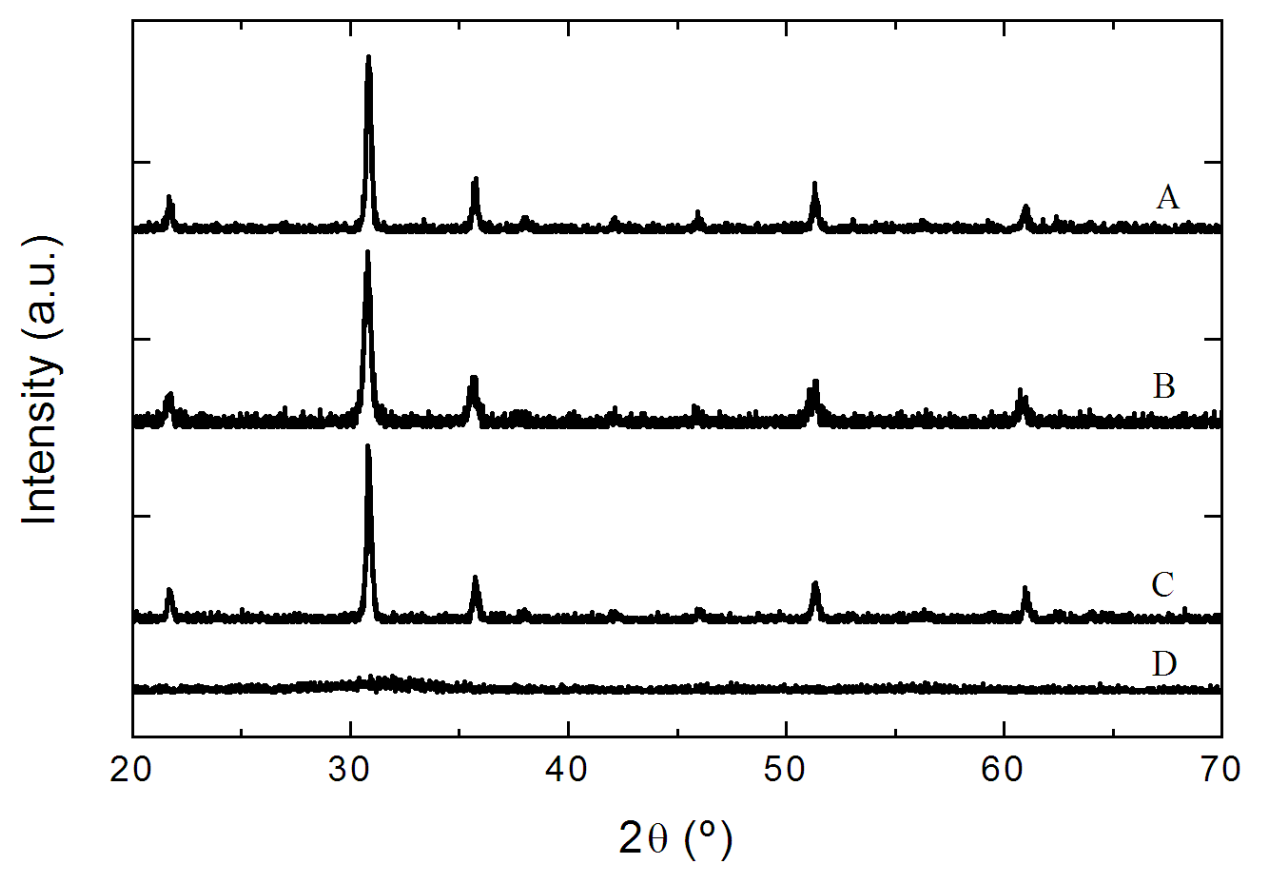


Fig. 3
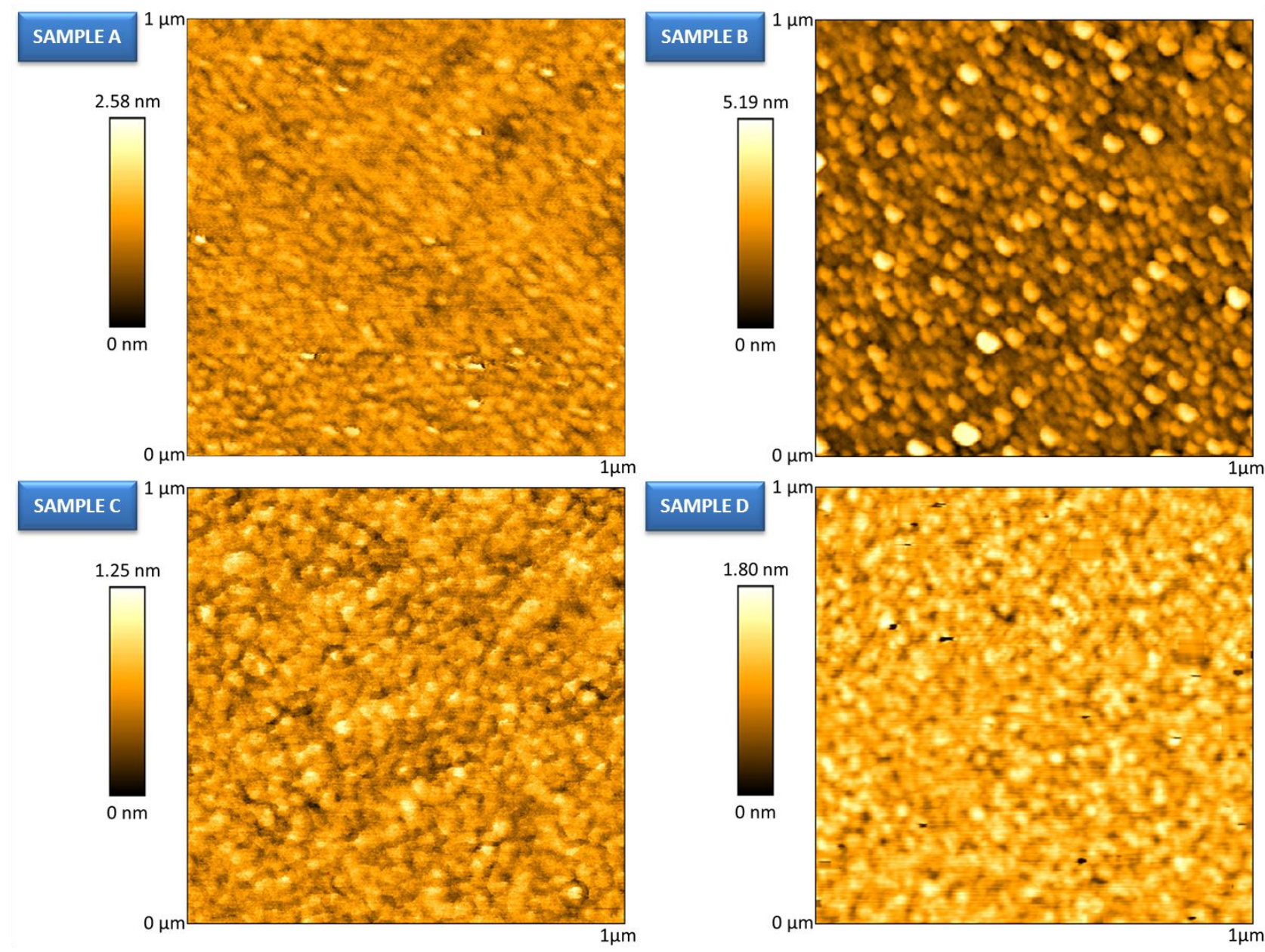
Fig. 4

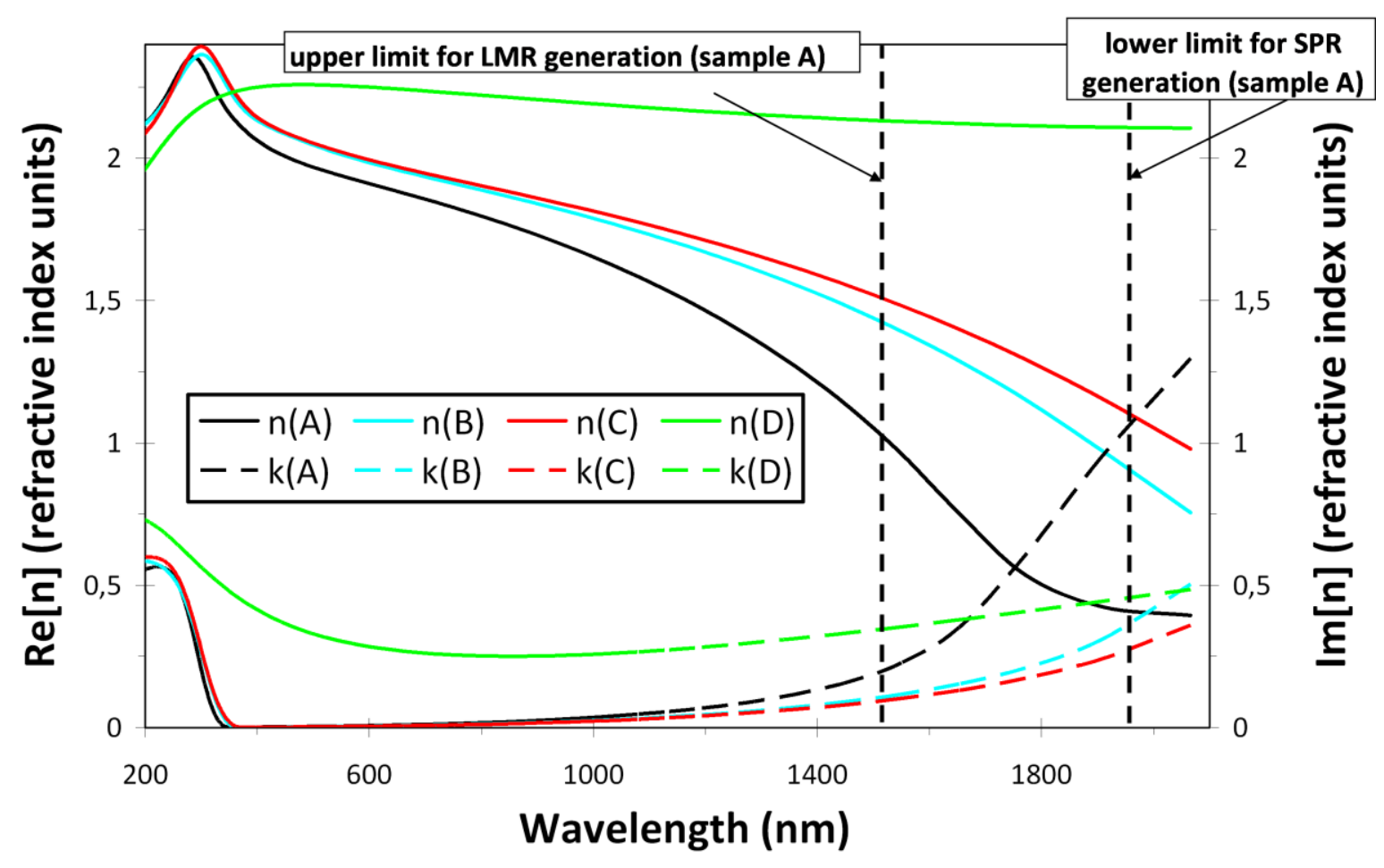


Fig. 5

a)
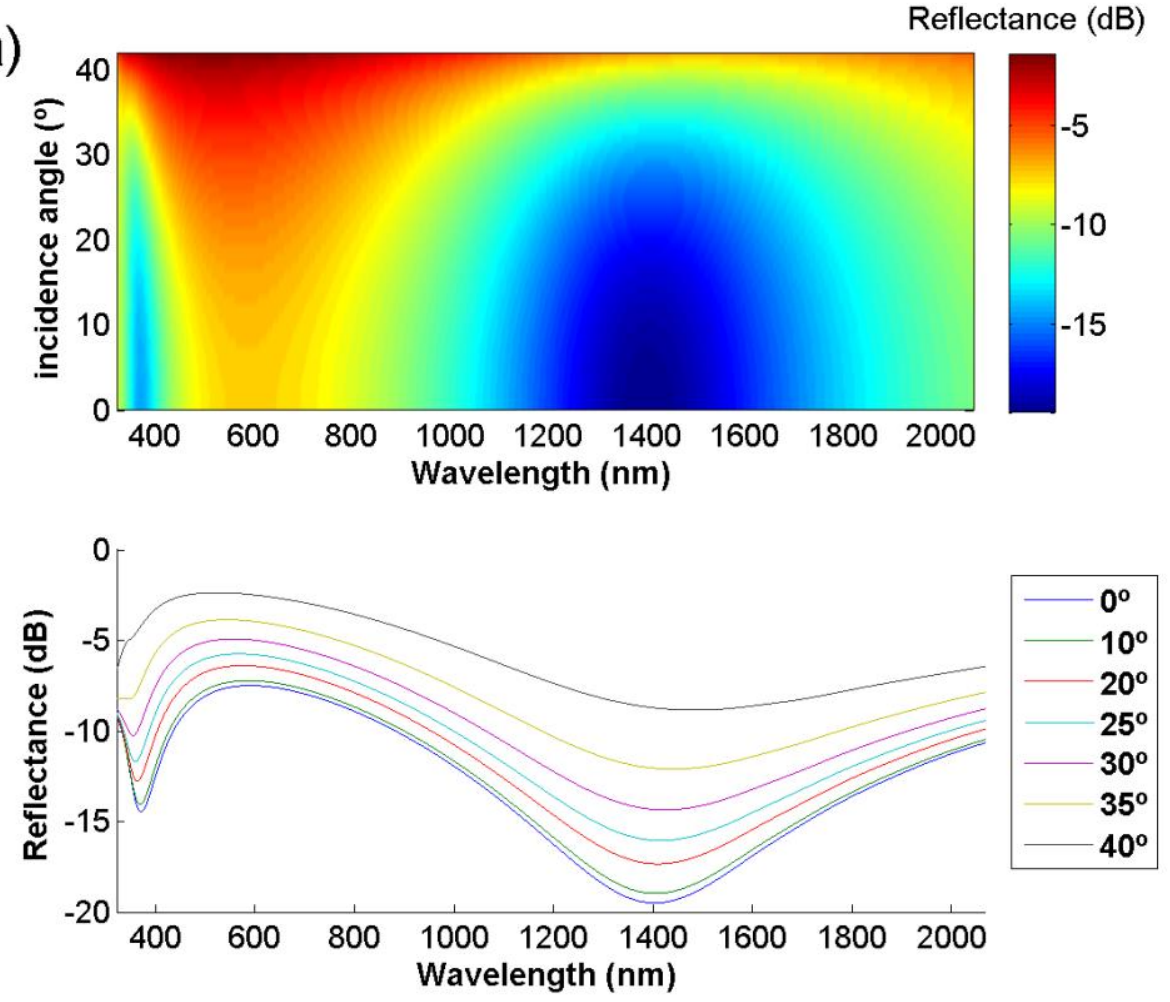

b)

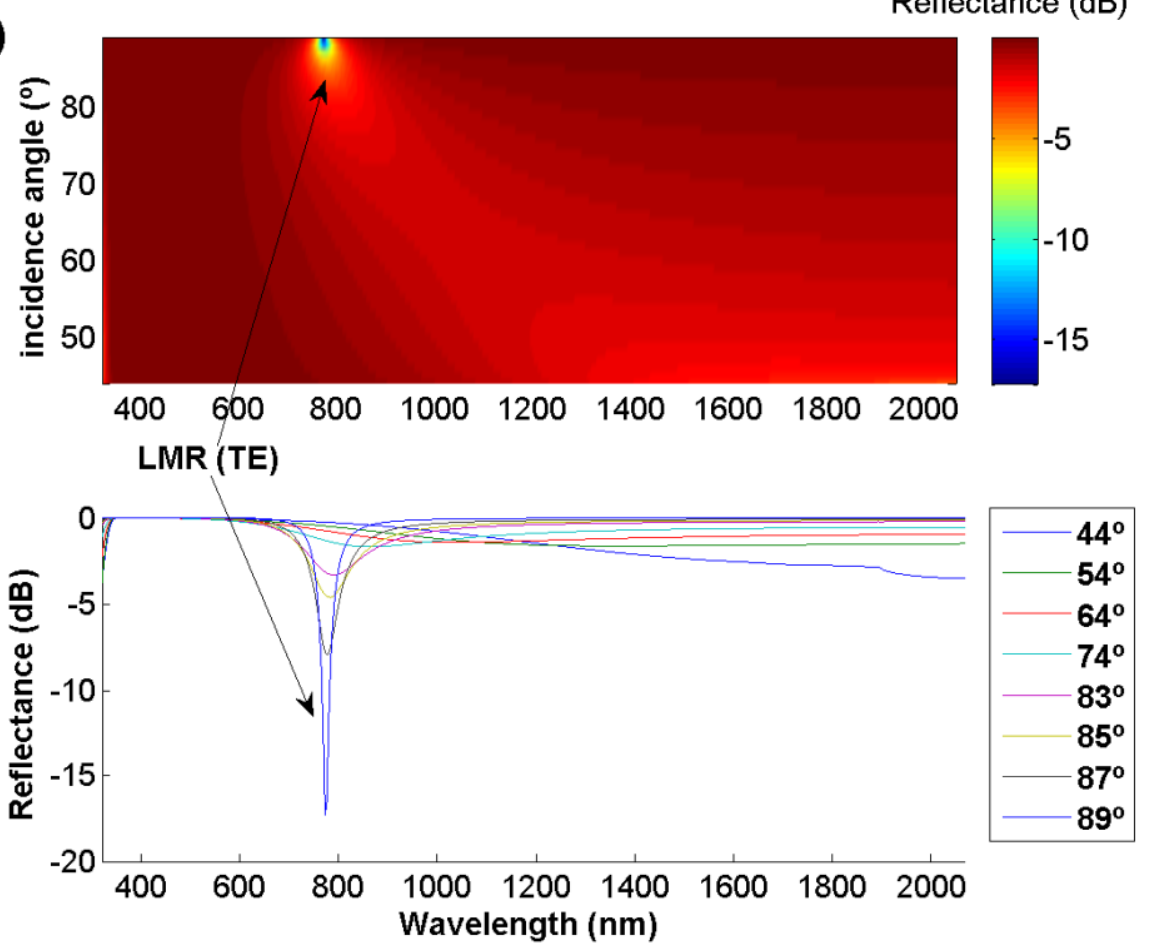


Fig. 6

a)
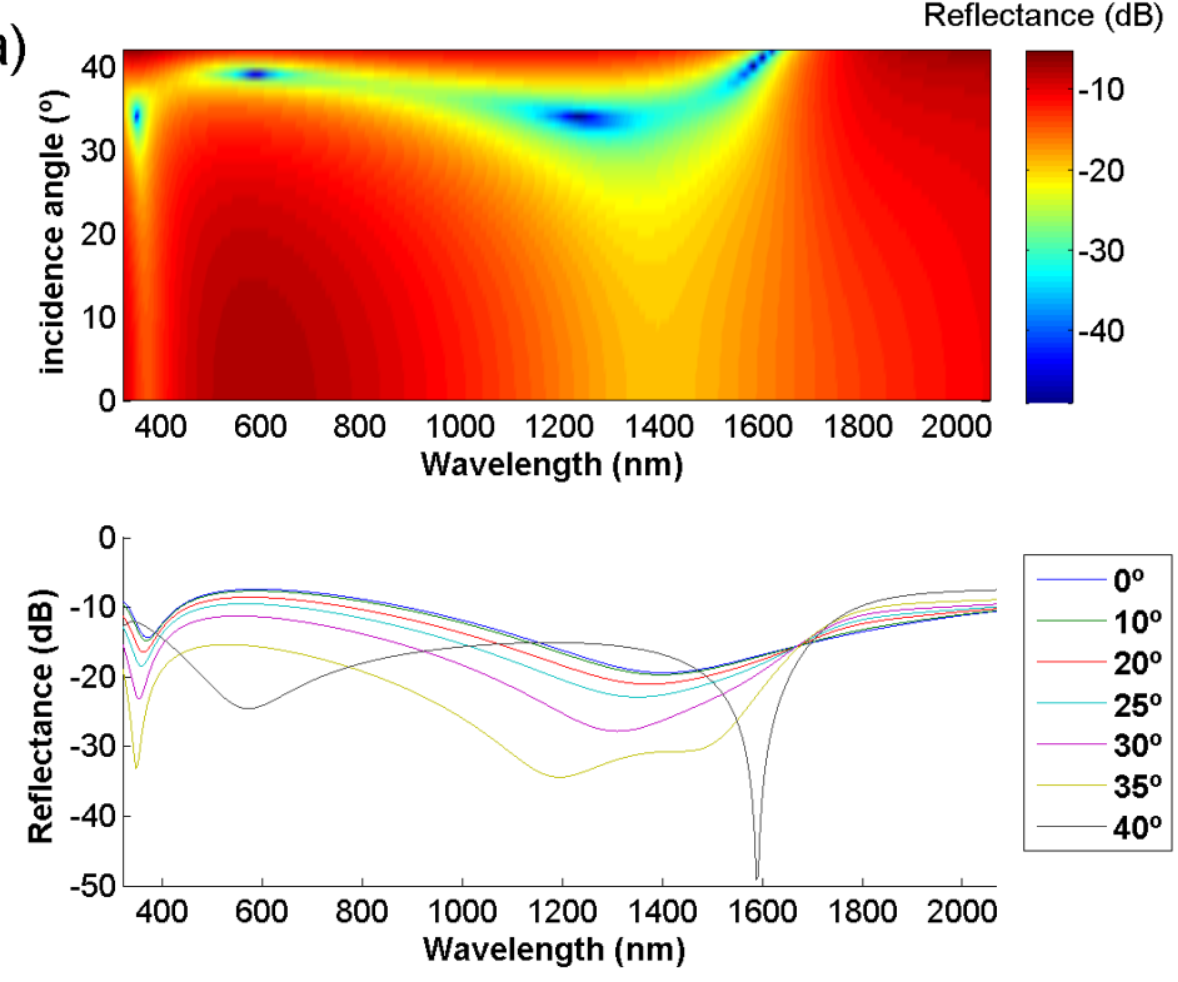

b)
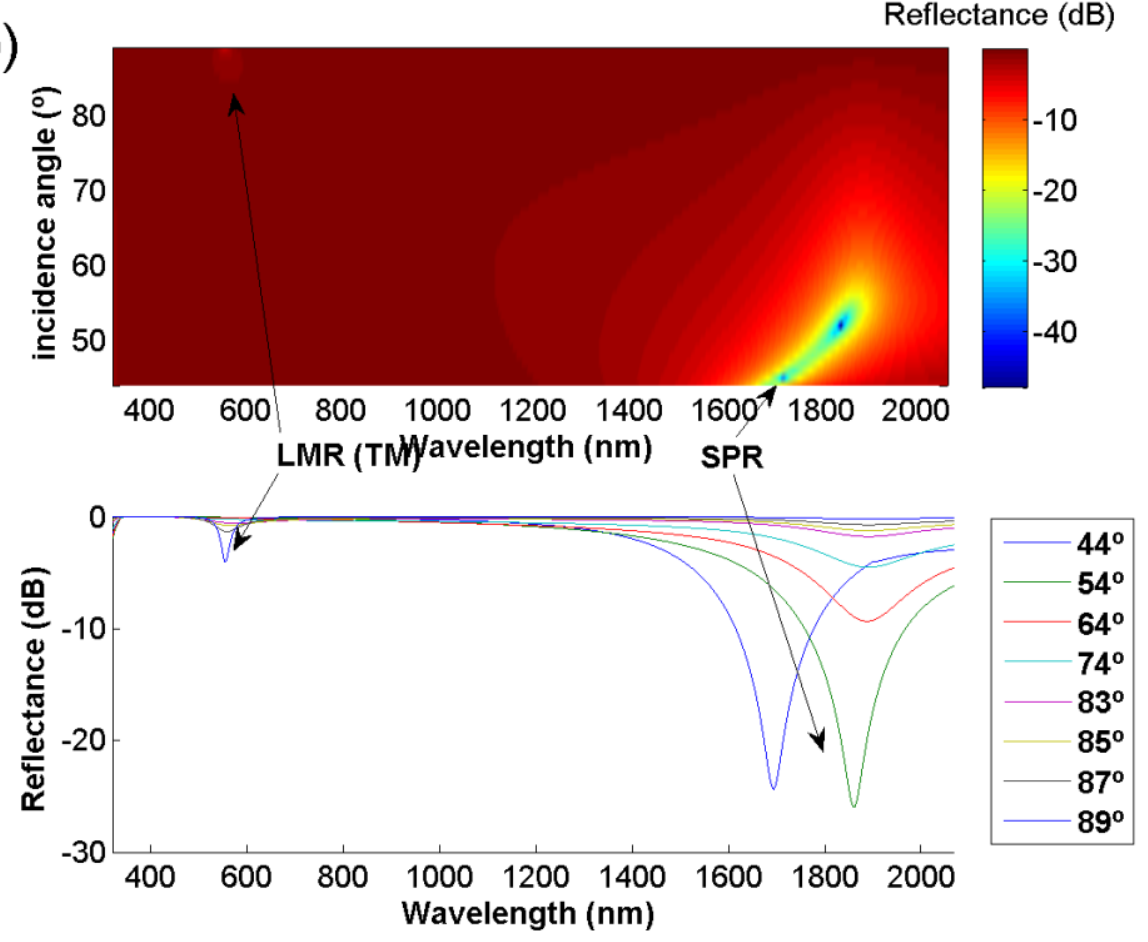
Fig. 7

a)
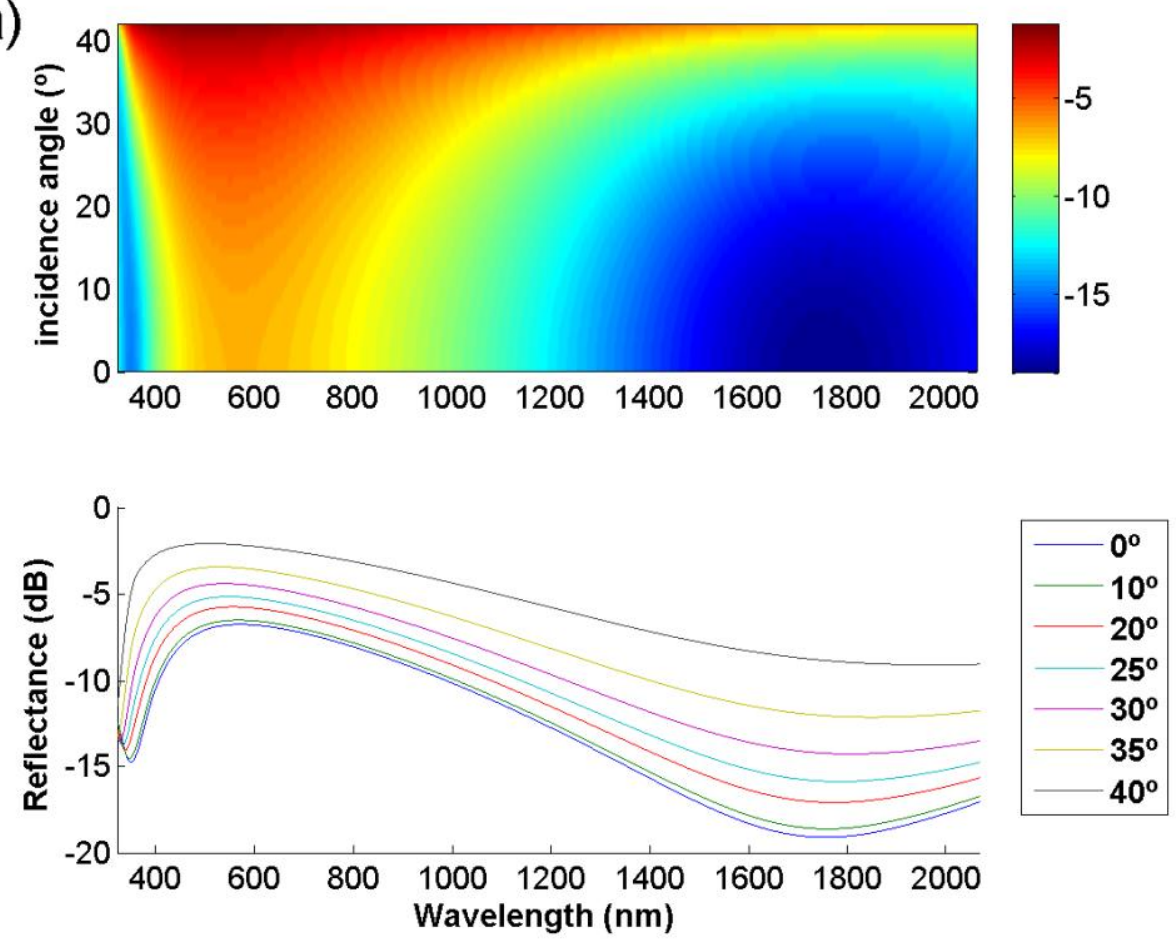

b)
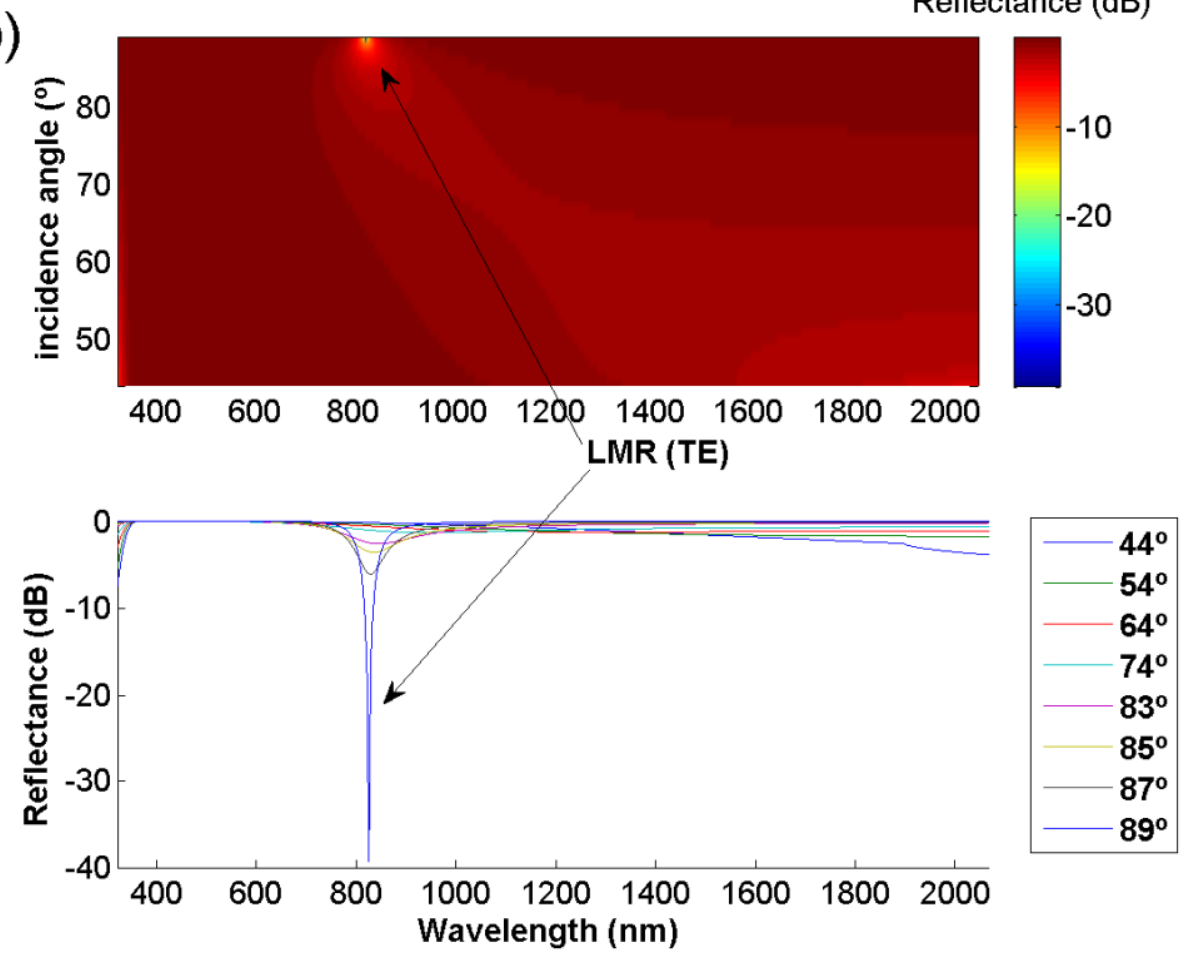
Fig. 8

a)
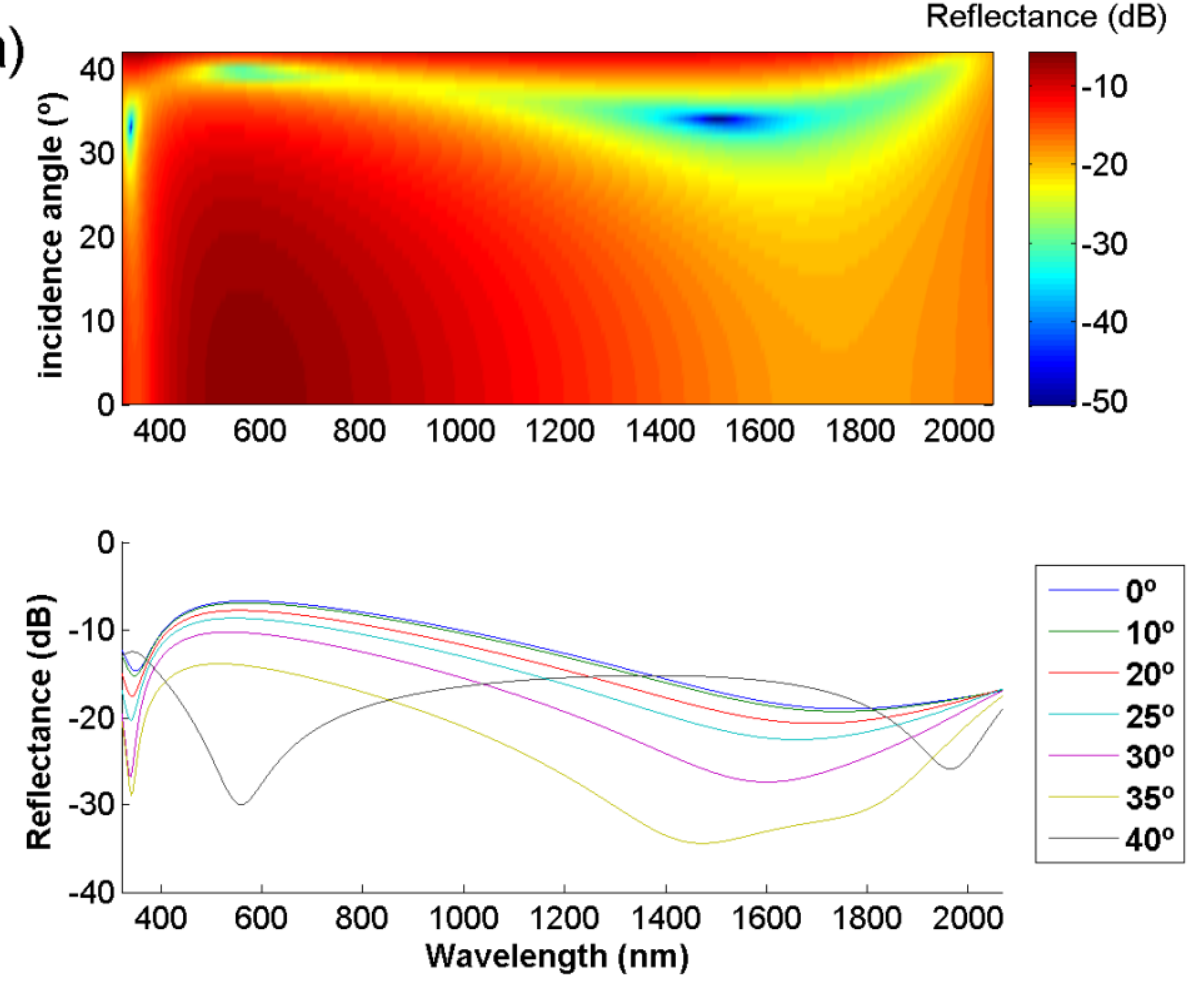

b)

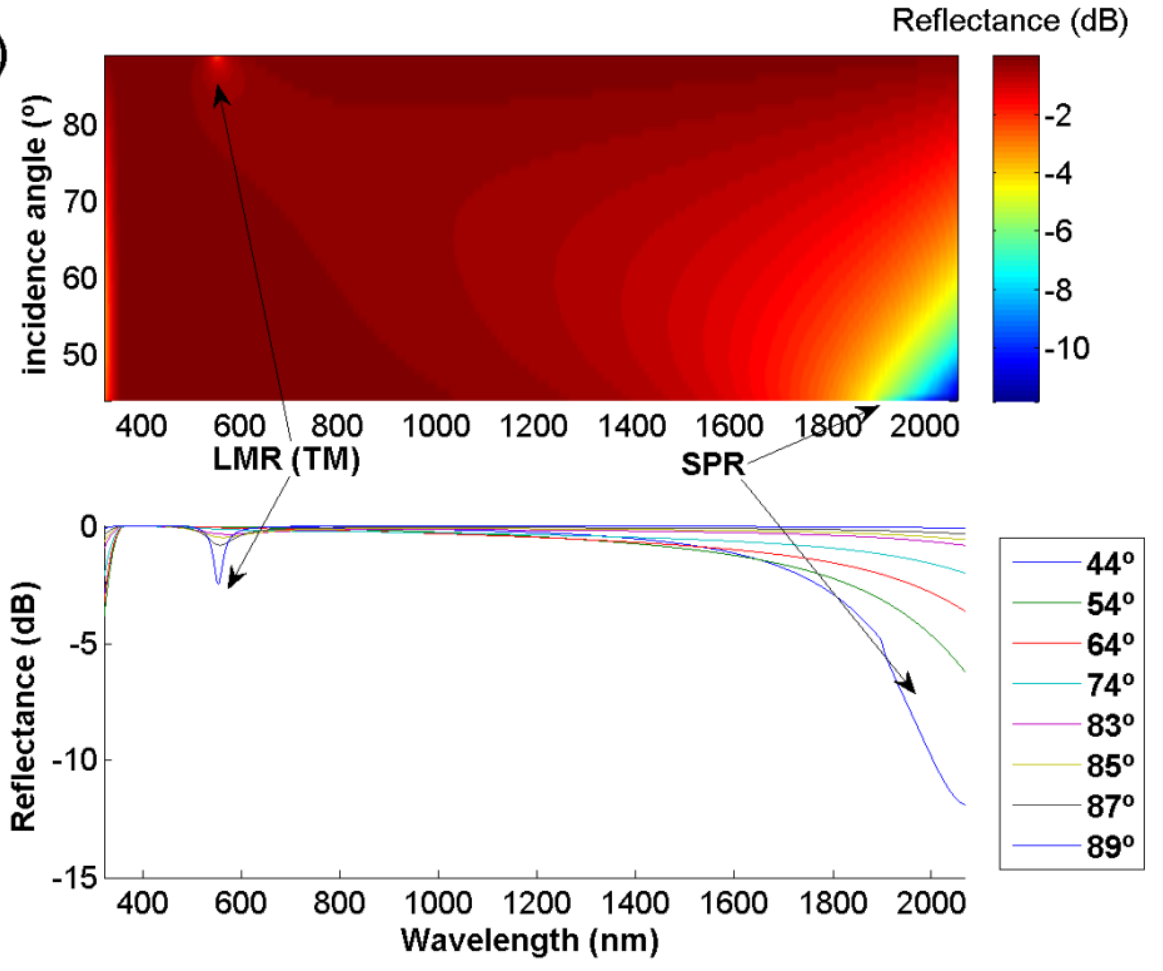

